Case report

\title{
A rare case of hepatic vanishing bile duct syndrome occurring after combination therapy with nivolumab and cabozantinib in a patient with renal carcinoma
}

Karim Gourari ${ }^{1} \ddagger$, Julien Catherine ${ }^{2,3}$, Soizic Garaud ${ }^{4}$, Joseph Kerger ${ }^{1}$, Antonia Lepida ${ }^{5}$, Georgala Aspasia ${ }^{1}$, Fabienne Lebrun', Maria Gomes Galdon' ${ }^{6}$, Thierry Gil ${ }^{1}$, Karen Willard-Gallo ${ }^{4}$ and Mireille Langouo Fontsa ${ }^{1,4 *}$

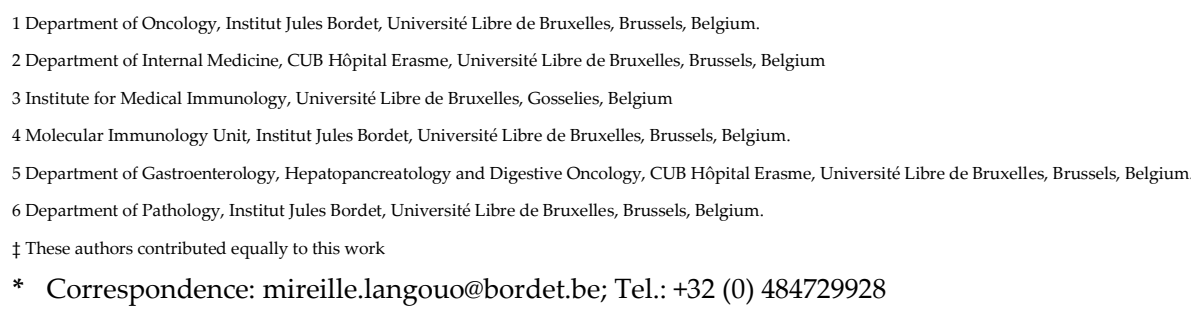

\begin{abstract}
Tyrosine kinase inhibitors (TKI) and immune checkpoint inhibitors (ICI) significantly improve the outcomes of patients with advanced clear cell renal cell carcinoma (ccRCC), but highgrade toxicities can occur, particularly during combination therapy. Herein, we report a patient with advanced metastatic ccRCC, who developed grade 4 cholestasis during combined therapy with nivolumab and cabozantinib. After the exclusion of common disorders associated with cholestasis and a failure of corticosteroids (CS), a liver biopsy was performed that demonstrated severe ductopenia. Consequently, a diagnosis of vanishing bile duct syndrome related to TKI and ICI administration was made, resulting in CS discontinuation and ursodeoxycholic acid administration. After a 7-months follow-up, liver tests had returned to normal values. Immunological studies revealed that our patient had developed robust T-cells and macrophages infiltrates in his lung metastasis, as well as in skin and liver tissues at the onset of toxicities. At the same time, peripheral blood immunophenotyping revealed significant changes in T-cell subsets suggesting their potential role in the pathophysiology of the disease.
\end{abstract}

Keywords: Checkpoint inhibitors; Immune related adverse events; Cholestasis; Severe ductopenia; Vanishing bile duct syndrome

\section{Introduction}

During the last decade, targeted therapies such as the new-generation tyrosine kinase inhibitors (TKI) and immune checkpoint inhibitors (ICI), have transformed the treatment of clear cell renal cell carcinoma (ccRCC) in the advanced stages of the disease[1-5]. Despite the excellent results observed with these drugs, a significant proportion of patients never benefits from treatment and most initial responders will ultimately develop progressive disease.

Another drawback is represented by the burden of added toxicity associated with combination strategy. Regarding overlapping toxicities, all vital organs can be affected, including the liver. In clinical trials, hepatitis was observed in up to $50 \%$ of patients during TKI treatment, but was generally transient and low grade, self-resolving without treatment discontinuation[6]. Severe hepatotoxicity also varies according to the VEFGR-TKI molecules (e.g. pazopanib, sunitinib)[6]. The management of such toxicities is often difficult, partially owing to a poor understanding of their underlying pathogenesis, particularly in the setting of dual therapy due to the overlapping spectra of toxicity[6, 7]. Safety 
data related to the concomitant use of ICI and TKI are issued from early phases of clinical trials or case reports, mostly corresponding to manageable toxicities which resolved after treatment discontinuation and/or administration of low dose corticosteroids (CS)[8].

Herein, we report a rare case of vanishing bile duct syndrome (VBDS) that arose after concomitant treatment with cabozantinib and nivolumab in the setting of an advanced metastatic ccRCC. A detailed assessment of the peripheral blood and tissue immune responses is then presented.

\section{Case Report}

A 58-year-old man with a history of mRCC was hospitalized in the setting of a pruritic cutaneous rash, grade IV cholestasis (total bilirubin: $28 \mathrm{mg} / \mathrm{dL}$, including $25 \mathrm{mg} / \mathrm{dL}$ conjugated; alkaline phosphatase: $805 \mathrm{UI} / \mathrm{L}$ ) and grade IV hepatitis (aspartate aminotransferase: $694 \mathrm{UI} / \mathrm{L}$; alanine amino-transferase: $1137 \mathrm{UI} / \mathrm{L}$ ). This occurred one month after the introduction of a TKI inhibitor (cabozantinib, $40 \mathrm{mg} /$ day) to his current treatment with nivolumab $(3 \mathrm{mg} / \mathrm{kg} / 3$ weeks) started in the context of metastatic progression 8 months before. There were no recent medication changes except the introduction of cabozantinib. Physical examination revealed a diffuse maculopapular skin eruption and conjunctival icterus, without any other sign of hepatic failure.

Blood analyses rapidly ruled out infectious, metabolic and autoimmune diseases. Ultrasound imaging and liver MRI excluded hepatic metastasis and obstructive biliary disease. A first liver biopsy was performed and showed images of centrolobular necrosis and cholangitis with poor mixed inflammation consisting of eosinophils and lymphocytes, consistent with a drug reaction (Figure 1, A-B). According to the presumed ICI-related adverse event (irAE) and/or TKI toxicity, nivolumab and cabozantinib were discontinued, and high dose CS were started (methylprednisolone, $2 \mathrm{mg} / \mathrm{kg} / \mathrm{d}$ ). Seven days later, no improvement in serum bilirubinaemia was observed, prompting the addition of oral mycophenolate mofetil (MMF, 1g/d) to CS according to ESMO guidelines [9]. Four weeks after dual immunosuppressive therapy and the lack of improvement in liver tests, CS were tapered to minimize side effects and a second biopsy was performed.

Remarkably, this second biopsy revealed a severe ductopenia with limited immune infiltrate within liver parenchyma, suggesting vanishing bile duct syndrome diagnosis (Figure 2). Accordingly, immunosuppressors were slowly tapered and ursodeoxycholic acid (UDCA) was started ( $5 \mathrm{mg} / \mathrm{kg} / \mathrm{d}$, with a progressive increase to $15 \mathrm{mg} / \mathrm{kg} / \mathrm{d}$ ). After 3 months, a slow decrease in serum conjugated bilirubin level was observed until its normalization at 7 months.

\section{Discussion}

There has been significant progress in the treatment of patients with mRCC, especially with the recent approval of TKI and immunotherapy combination as first-line treatment. In 2019, the FDA approved pembrolizumab (anti-PD-1) plus axitinib therapy, the combo demonstrating survival advantages compared to sunitinib[10]. Since then, the combination of avelumab (anti-PD-L1) with axitinib has also been compared to sunitinib and finally approved (survival data are still immature; follow-up for the final analysis is ongoing)[11] . Despite their efficacy, the major concerns associated with these dual therapies are the potential cumulated and/or overlapping toxicities.

(Case)

Here we report an exceptional hepatic manifestation, namely a vanishing bile duct syndrome, in a patient receiving cabozantinib and nivolumab for metastatic progression of a mRCC. VBDS is a rare condition in which patients develop chronic cholestasis associated with ductopenia, defined as loss of interlobular bile ducts in $>50 \%$ of portal areas 
on histological examination[12]. Causes of ductopenia are diverse and include drugs (e.g., antibiotics, anticonvulsants), immune disorders (e.g., primary biliary cholangitis), infectious diseases (EBV, CMV) and haematological malignancies (Hodgkin lymphoma)[12] . To our knowledge, this case represents the first VBDS occurring during VEGFR-TKI/ICI combination to date, although Zhong and al. reported the onset of a fatal VBDS 25 days after pazopanib introduction, directly succeeding pembrolizumab therapy[13]. In our case, the recent initiation of cabozantinib suggests its potential causative role although grade IV cholestasis seems rare and often explained by other factors during VEGFR-TKI therapy (e.g., hepatic insufficiency associated with hepatocellular carcinoma) [6, 14, 15].

On the other hand, ICI have been associated with various types of liver impairment, including marked cholestatic hepatitis with recent case reports describing steroid and MMF therapy-resistant disease where the biliary tract seems the major target of injury, even if hepatocellular damages are also present $[16,17]$. Moreover, three cases of VBDS occurred in patients receiving ICI alone while no reports of TKI-related VBDS could be retrieved from the literature $[7,18,19]$. Table 1 summarizes the main clinical features of those patients. Briefly, VBDS was always associated with pembrolizumab use, appeared after an a single injection in two cases and induced a mean elevation of conjugated bilirubinemia of $26,3 \mathrm{mg} / \mathrm{dL}$. The ICI was discontinued in all patients, and CS, MMF and UDCA were used in two cases while the remaining patient declined any therapy. Surprisingly, this last patient normalized his hepatic tests after 16 weeks, while another required CS and UDCA to achieve the same outcome. The third patient was ultimately treated by plasmapheresis and died 26 days after cholestasis onset. These observations, including ours, suggest that ductal loss leading to VBDS could explain why immunosuppressive therapy could be ineffective once ductal damages appear. In our case, whether cabozantinib had enhanced the immune response especially towards ductal tissue or impaired cellular regeneration after immune destruction is unknown, but could explain the timescale between VEGFR-TKI initiation and cholestasis occurrence.

\section{(Biomarkers)}

Given the unique features of this case and the absence of any relevant pathophysiologic mechanism underlying VBDS during ICI/VEGFR-TKI therapy, we decided to assess local immune responses occurring in our patient at the onset of adverse events. First, we studied tissue immune markers in lung metastasis at disease progression, and in skin and liver tissues at the onset of irAEs using multiplex immunohistochestry (mIHC) on Formalin-fixed and paraffin-embedded (FFPE) tissue blocks coming from biopsies from lung metastasis (January 2019), skin eruption (November 2019), and liver at the onset of the irAES (November 2019 and January 2020). Description tissue immunophenotyping using mIHC is presented in Supplementary Figure S1.

Liver biopsies were taken before (PRE) and on $(\mathrm{ON})$ corticosteroid treatment (Figure 3 , A). We measured the relative amount (percentage in total cells) of CD4+, CD8+ T cells, CD20+ B cells, FOXP3+ regulatory $\mathrm{T}$ cells (Treg), and CD68+ macrophages within the stroma and the epithelium area of each tissue. All tissues showed immune cell infiltration including CD4+, CD8+ T cells, and macrophages, both in the stroma and epithelium areas (Figure 3, B). B cells and Treg were less present. Interestingly, $\mathrm{T}$ cell infiltration in liver was reduced by corticosteroid treatment, both CD4+ $(15 \%$ and $3 \%$ of total cells in the stroma, respectively) and CD8+ (27\% and $7 \%$ of total cells in the stroma, respectively) $\mathrm{T}$ cells. CD68+ macrophages were not impacted by corticosteroid treatment in liver.

In line with these observations, robust T-cell infiltration, activation, and clonal expansion were already described in cardiac and skeletal muscle of two patients with melanoma in whom fatal myocarditis developed after treatment with ipilimumab and nivolumab[20]. Further studies of $\mathrm{T}$ cells reactivity within tumor microenvironment are needed to elucidate their contribution in toxicities.

Metastatic ccRCC patients treated with nivolumab from the CheckMate-010 trial revealed that high percentage of CD8+ tumor infiltrating lymphocytes (TIL) that are PD- 
1+TIM-3-LAG-3- within tumor microenvironment (assessed by mIHC) correlated with high levels of T-cell activation, including cytolytic activity, effector T-cells and IFN $\gamma$ response, and was associated with longer median immune-related PFS and higher odds ratio rate on nivolumab [21]. However, CyTOF analyses on clinical samples from melanoma patients treated with ICI didn't show any association between the frequency of PD-1 expression on either CD4+ or CD8+ T cells with clinical benefit[22]. In the current study, the presence of PD1+ T cells were not assessed by $\mathrm{mIHC}$, however increase of PD1+ CD4+ and CD8+ $\mathrm{T}$ cell frequencies was observed in the PB on CS therapy at the onset of toxicities. Further analyses are required to understand whether the expression of PD-1 reflects T cell exhaustion/dysfunction or activation of anti-tumor response and whether these cells could be responsible for hepatic duct injury in this setting of ICI/TKI-induced VBDS.

\section{Conclusions}

Given overlapping hepatic toxicities of TKI and ICI, severe liver impairment can occur with a delayed management because of the poor understanding of the underlying pathogenesis. Although data concerning toxicity and management of each drug used as monotherapy are more and more available, the current lack of knowledge regarding potential toxicity of their combined administration should prompt thorough evaluation including liver biopsy, case reporting and translational research. Particularly, the occurrence of immunosuppressor-resistant cholestatic hepatitis should raise suspicion for vanishing bile duct syndrome in patients treated with TKI/ICI bitherapy.

Author Contributions: AG, FL, JC, MLF and TG managed the patient. AL was the expert consultant in hepatology. MGG performed the histopathological diagnosis. SG and KWG performed the immunological analysis. JC, JK, KG, MLF and SG wrote the manuscript. All authors have read and agreed to the published version of the manuscript.

Funding: No funding was specifically dedicated to this study. JC is supported by the National Fund for Scientific Research (F.R.S-FNRS) as research fellow (grant: 1/A/094/21f).

Institutional Review Board Statement: Not applicable.

Informed Consent Statement: Not applicable.

Acknowledgments: The authors thank Sandrine Aspelagh for scientific discussions. Anaïs Boissons for her experimental help for the multiplex immunohistochemistry staining.

Conflicts of Interest: The authors declare no conflict of interest.

\section{References}

1. Choueiri, T.K., et al., Cabozantinib Versus Sunitinib As Initial Targeted Therapy for Patients With Metastatic Renal Cell Carcinoma of Poor or Intermediate Risk: The Alliance A031203 CABOSUN Trial. J Clin Oncol, 2017. 35(6): p. 591-597.

2. Choueiri, T.K., et al., Cabozantinib versus sunitinib as initial therapy for metastatic renal cell carcinoma of intermediate or poor risk (Alliance A031203 CABOSUN randomised trial): Progression-free survival by independent review and overall survival update. Eur J Cancer, 2018. 94: p. 115-125. 
3. Rini, B.I., et al., The society for immunotherapy of cancer consensus statement on immunotherapy for the treatment of advanced renal cell carcinoma (RCC). J Immunother Cancer, 2019. 7(1): p. 354.

4. $\quad$ Rodriguez-Vida, A., et al., New treatment options for metastatic renal cell carcinoma. ESMO Open, 2017. 2(2): p. e000185.

5. Makhov, P., et al., Resistance to Systemic Therapies in Clear Cell Renal Cell Carcinoma: Mechanisms and Management Strategies. Mol Cancer Ther, 2018. 17(7): p. 1355-1364.

6. Shah, R.R., J. Morganroth, and D.R. Shah, Hepatotoxicity of tyrosine kinase inhibitors: clinical and regulatory perspectives. Drug Saf, 2013. 36(7): p. 491-503.

7. Doherty, G.J., et al., Severe steroid-resistant anti-PD1 T-cell checkpoint inhibitor-induced hepatotoxicity driven by biliary injury. ESMO Open, 2017. 2(4): p. e000268.

8. Gao, L., et al., Adverse Events of Concurrent Immune Checkpoint Inhibitors and Antiangiogenic Agents: A Systematic Review. Front Pharmacol, 2019. 10: p. 1173.

9. Haanen, J., et al., Management of toxicities from immunotherapy: ESMO Clinical Practice Guidelines for diagnosis, treatment and follow-up. Ann Oncol, 2017. 28(suppl_4): p. iv119-iv142.

10. Rini, B.I., et al., Pembrolizumab plus Axitinib versus Sunitinib for Advanced Renal-Cell Carcinoma. N Engl J Med, 2019. 380(12): p. 1116-1127.

11. Tomita, Y., et al., Efficacy of avelumab plus axitinib $(A+A x)$ versus sunitinib (S) by number of IMDC risk factors and tumor sites at baseline in advanced renal cell carcinoma (aRCC): Extended follow-up results from JAVELIN Renal 101. Journal of Clinical Oncology, 2021.39(6_suppl): p. 302-302.

12. Reau, N.S. and D.M. Jensen, Vanishing bile duct syndrome. Clin Liver Dis, 2008. 12(1): p. 203-17, x.

13. Zhong, Y.Y., et al., Vanishing bile duct syndrome associated with pazopanib after progression on pembrolizumab. Can J Urol, 2020. 27(4): p. 10339-10341.

14. Elisei, R., et al., Cabozantinib in progressive medullary thyroid cancer. J Clin Oncol, 2013. 31(29): p. $3639-46$.

15. Ghatalia, P., et al., Hepatotoxicity with vascular endothelial growth factor receptor tyrosine kinase inhibitors: A meta-analysis of randomized clinical trials. Crit Rev Oncol Hematol, 2015. 93(3): p. 257-76.

16. Matsubara, T., et al., Nivolumab Induces Sustained Liver Injury in a Patient with Malignant Melanoma. Intern Med, 2018. 57(12): p. 1789-1792.

17. Reddy, H.G., B.J. Schneider, and A.W. Tai, Immune Checkpoint Inhibitor-Associated Colitis and Hepatitis. Clin Transl Gastroenterol, 2018. 9(9): p. 180.

18. Thorsteinsdottir, T., et al., Fatal Cholestatic Liver Injury during Treatment with PD1 Immune Checkpoint Inhibitor for Malignant Melanoma: A Case Report. Case reports in oncology, 2020. 13(2): p. 659-663.

19. Masetti, C., et al., Pembrolizumab-Induced Vanishing Bile Duct Syndrome: a Case Report. SN Comprehensive Clinical Medicine, 2021. 3(3): p. 906-908.

20. Johnson, D.B., et al., Fulminant Myocarditis with Combination Immune Checkpoint Blockade. N Engl J Med, 2016. 375(18): p. 17491755.

21. Pignon, J.C., et al., irRECIST for the Evaluation of Candidate Biomarkers of Response to Nivolumab in Metastatic Clear Cell Renal Cell Carcinoma: Analysis of a Phase II Prospective Clinical Trial. Clin Cancer Res, 2019. 25(7): p. 2174-2184.

22. Subrahmanyam, P.B., et al., Distinct predictive biomarker candidates for response to anti-CTLA-4 and anti-PD-1 immunotherapy in melanoma patients. J Immunother Cancer, 2018. 6(1): p. 18. 

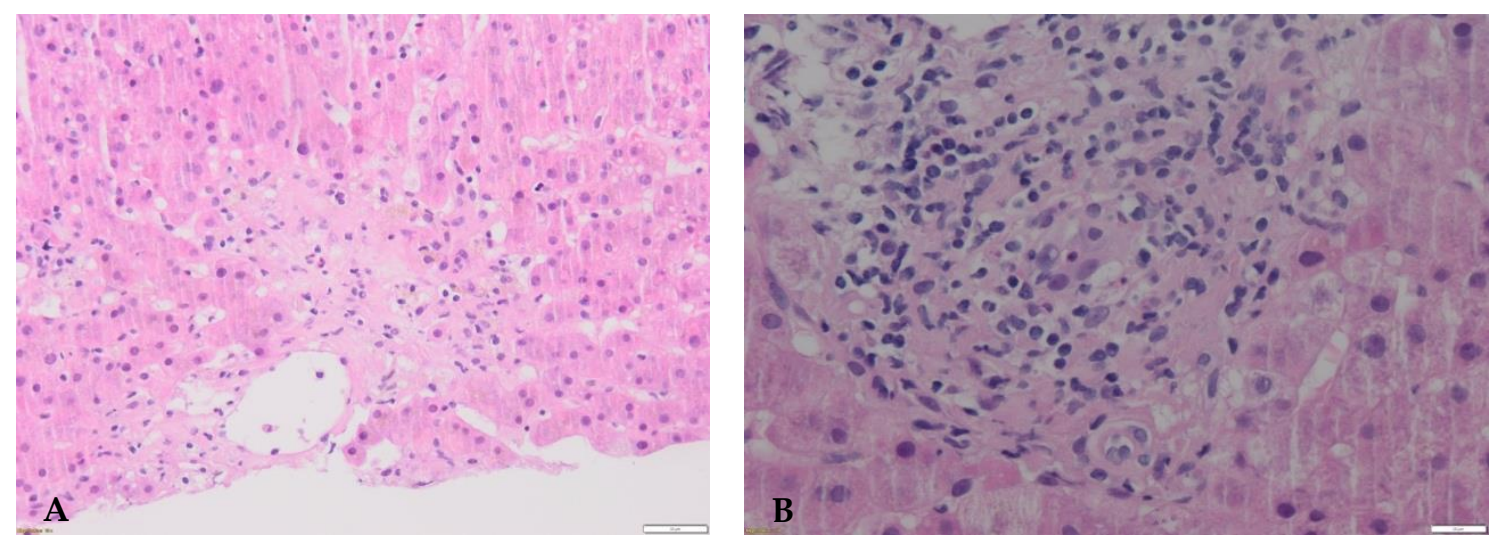

Figure 1. First liver biopsy performed at presentation. Hematoxylin and eosin-stained sections. A: Liver parenchyma is characterized by centrolobular necrosis, sometimes confluent. B: Immune infiltrate predominates around portal areas and is mainly composed of lymphocytes and rare eosinophils.

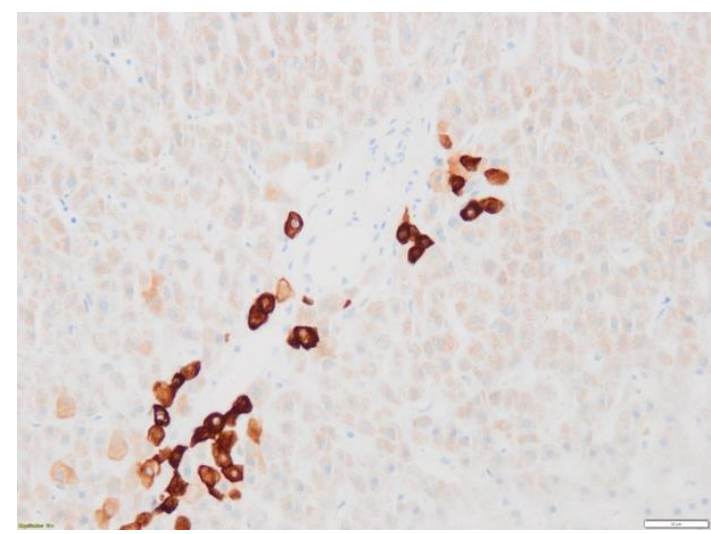

Figure 2. Second liver biopsy performed 5 weeks after cholestasis onset. Immunohistochemical staining with anti-cytokeratin7 (CK7) antibody. Large interlobular bile ducts are absent as anti-CK7 staining only reveal ductular reaction. These findings are suggestive of ductopenia. 
Figure 3

A.
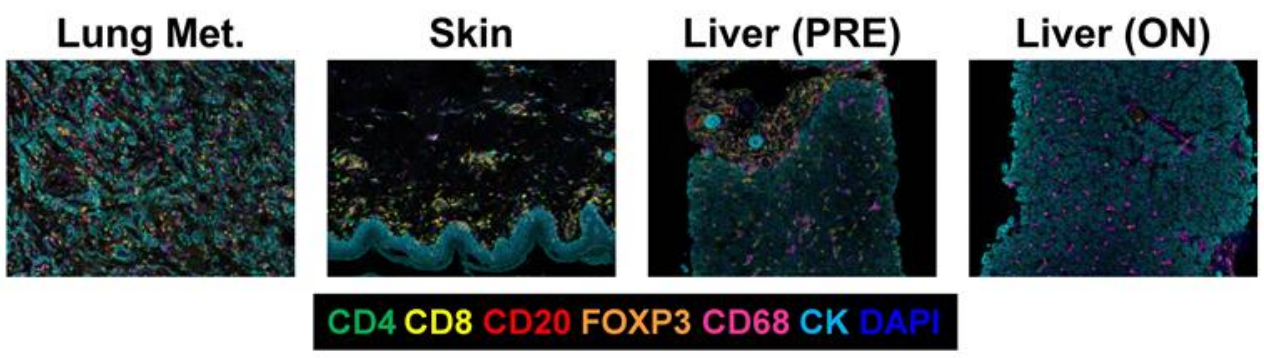

B.
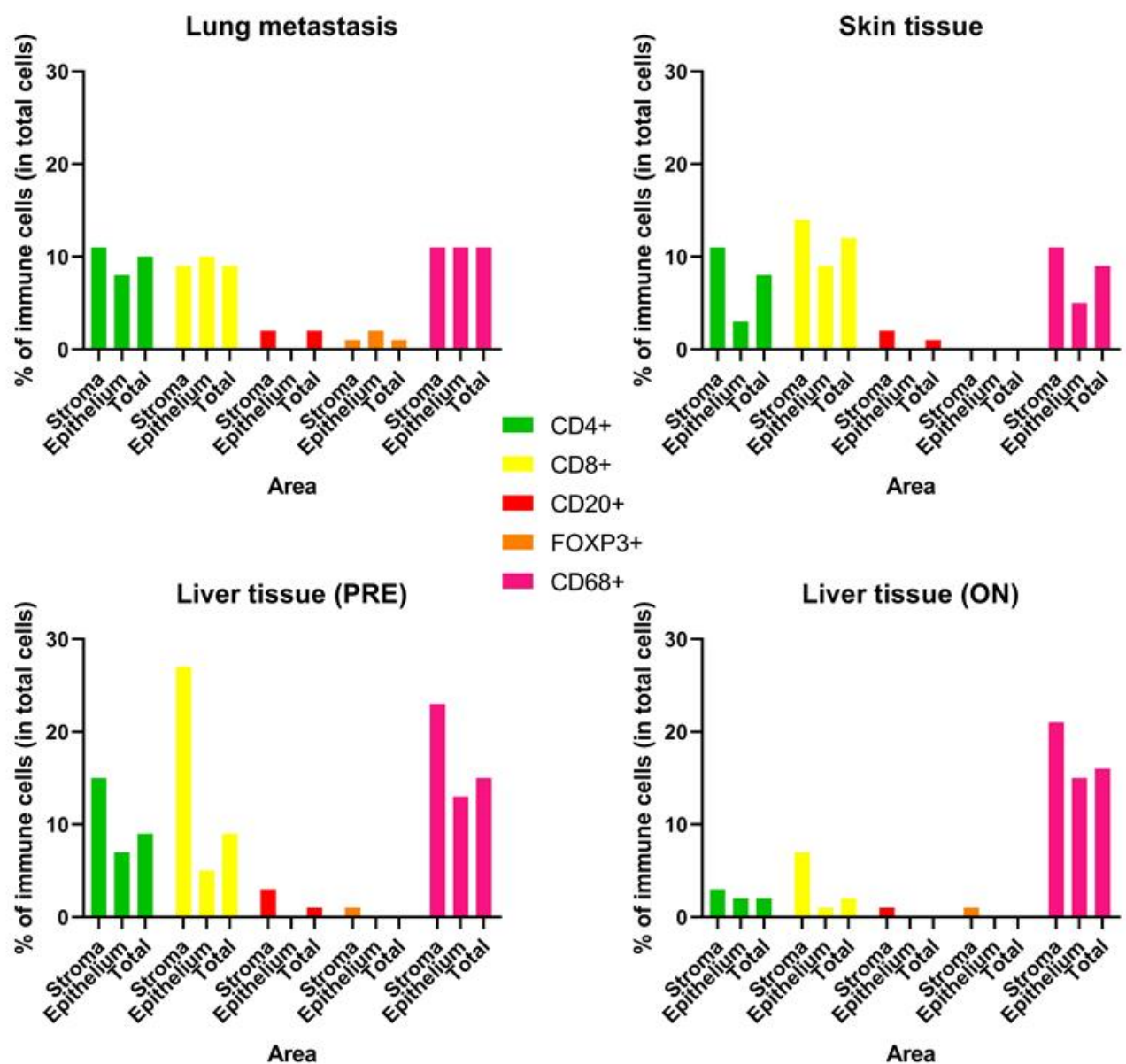

1. Figure 3: Tissue immune infiltration at disease progression and at the onset of toxicities.

A. Representative fluorescent multiplexed IHC images of lung metastasis, skin tissue and liver tissues before (PRE) and on $(\mathrm{ON})$ corticosteroid treatment for both first and second biopsy, and MMF only for the second biopsy. Tumor-infiltrating lymphocytes were revealed by CD20 (red), CD8 (yellow) and CD4 (green), FOXP3 (blue), CD68 (magenta), and tumor cells by panCK (cyan).

B. Quantification of immune cell as percent of total cells within the stroma, the epithelium, and total area of each tissue 
Table 1. Published cases of vanishing bile duct syndromes occurring during immune checkpoint inhibitor and/or tyrosine kinase inhibitor therapy.

\begin{tabular}{|c|c|c|c|c|c|c|c|c|}
\hline $\begin{array}{l}\text { Reference } \\
10 \\
11 \\
12\end{array}$ & Neoplasia & $\begin{array}{l}\text { Suspected } \\
\text { causal therapy }\end{array}$ & $\begin{array}{l}\text { Time-to- } \\
\text { onset }\end{array}$ & $\begin{array}{l}\text { Peak c- } \\
\text { bilirubin / } \\
\text { ALAT }\end{array}$ & $\begin{array}{l}\text { Signs of } \\
\text { hepatic } \\
\text { failure }\end{array}$ & Therapy & Issue & \\
\hline $\begin{array}{l}\text { Doherty, } 2017 \\
\text { (ref) }\end{array}$ & Melanoma & Pembrolizumab & $\begin{array}{l}1 \text { cycle } \\
(8 d)\end{array}$ & $\begin{array}{l}23 \mathrm{mg} / \mathrm{dL} \\
1536 \mathrm{IU} / \mathrm{L}\end{array}$ & Absent & $\begin{array}{l}\mathrm{CS} \quad 1 \mathrm{~m} / \mathrm{kg} \\
\mathrm{MMF}, \mathrm{UDCA}\end{array}$ & $\begin{array}{l}\text { Normalized c-bili } \\
\text { under CS and } \\
\text { UDCA therapy }\end{array}$ & \\
\hline $\begin{array}{l}\text { Thorsteinsdottir, } \\
2020 \text { (ref) } \\
20 \\
21 \\
22\end{array}$ & Melanoma & Pembrolizumab & 12 cycles & $\begin{array}{l}32 \mathrm{mg} / \mathrm{dL} \\
450 \mathrm{IU} / \mathrm{L}\end{array}$ & Absent & $\begin{array}{l}\text { CS } 2 \mathrm{mg} / \mathrm{kg} \\
\text { MMF, UDCA, } \\
\text { plasmaphere- } \\
\text { sis }\end{array}$ & $\begin{array}{l}\text { Death }(\mathrm{d}+26 \text { after } \\
\text { cholestasis onset) }\end{array}$ & \\
\hline $\begin{array}{l}\text { Zhong, } 2020 \\
\text { (ref) }\end{array}$ & $\mathrm{mRCC}$ & $\begin{array}{l}\text { Pembrolizumab } \\
\text { (previously) } \\
\text { Pazopanib (on- } \\
\text { going) }\end{array}$ & $\begin{array}{l}5 \text { cycles } \\
(25 d)\end{array}$ & $\begin{array}{l}22 \mathrm{mg} / \mathrm{dL} \\
800 \mathrm{IU} / \mathrm{L}\end{array}$ & Absent & $\begin{array}{l}\text { CS } 2 \mathrm{mg} / \mathrm{kg} \\
\text { UDCA }\end{array}$ & $\begin{array}{l}\text { Death }(\mathrm{d}+30 \text { after } \\
\text { cholestasis onset) }\end{array}$ & \\
\hline $\begin{array}{l}\text { Bdasetti, } 2021 \\
\text { (Pef) }\end{array}$ & NSCLC & Pembrolizumab & $\begin{array}{l}1 \text { cycle } \\
(21 d)\end{array}$ & $\begin{array}{l}24 \mathrm{mg} / \mathrm{dL} \\
818 \mathrm{IU} / \mathrm{L}\end{array}$ & Absent & $\begin{array}{l}\text { None (patient } \\
\text { refusal) }\end{array}$ & $\begin{array}{l}\text { Normalized c-bili } \\
\text { after } 16 \text { weeks }\end{array}$ & \\
\hline Present case & $\mathrm{mRCC}$ & $\begin{array}{l}\text { Nivolumab, } \\
\text { Cabozantinib } \\
\text { (both ongoing) }\end{array}$ & $\begin{array}{l}6 \text { cycles } \\
(28 \mathrm{~d})\end{array}$ & $\begin{array}{l}28 \mathrm{mg} / \mathrm{dL} \\
1137 \mathrm{IU} / \mathrm{L}\end{array}$ & Absent & $\begin{array}{l}\mathrm{CS} 2 \mathrm{mg} / \mathrm{kg} \\
\mathrm{MMF}, \mathrm{UDCA}\end{array}$ & $\begin{array}{l}\text { Normalized c-bili } \\
\text { after } 28 \text { weeks un- } \\
\text { der UDCA therapy }\end{array}$ & $\begin{array}{l}\text { Abbreviations: } \\
\text { ALAT, alanine } \\
\text { aminotransfer- }\end{array}$ \\
\hline
\end{tabular}




\section{Supplementary material}

Tissue immunophenotyping

Four-micrometer sections from full FFPE blocks were used for manual multiplex immunohistochemistry (mIHC) staining according to the manufacturer's instructions (Opal 7 Solid Tumor Immunology kit, Akoya Biosciences®).

The used antibodies were CD4 (80 ng/ml, 90 min, Akoya Biosciences $\left.{ }^{\circledR}\right)$, CD8 (68 ng/ml, 90 min, Akoya Biosciences $\left.{ }^{\circledR}\right)$, CD20 (45 $\mathrm{ng} / \mathrm{ml}, 90 \mathrm{~min}$, Akoya Biosciences $\left.{ }^{\circledR}\right)$, FOXP3 (674 ng/ml, $90 \mathrm{~min}$, Akoya Biosciences $\left.{ }^{\circledR}\right)$, CD68 (16 ng/ml, 90 min, Akoya Biosciences $\left.{ }^{\circledR}\right)$, and panCK $\left(400 \mathrm{ng} / \mathrm{ml}, 90 \mathrm{~min}\right.$, Akoya Biosciences $\left.{ }^{\circledR}\right)$.

Slides were mounted with Vectashield Hardset Antifade Mounting Medium (VectorLaboratories). Multiplexed slides were scanned on Vectra Polaris Automated Quantitative Pathology Imaging System (Akoya Biosciences $\left.{ }^{\circledR}\right)$. InForm ${ }^{\circledR}$ Tissue Finder ${ }^{\mathrm{TM}}$ software was used in order to deconvolute the multispectral images, to segment issue, and to segment and phenotype cells. The percentage of cells was calculated on full section except for lung metastasis for which $25 \%$ of the section was multispectrally acquired. Digital quantification was then performed using PhenoptrReports (Akoya Biosciences®). GraphPad Prism8 software was used to graph individual datapoint. 